\title{
In vitro fermentation and prebiotic potential of pigeon pea (Cajanus cajan (L.) Millsp.) flour
}

\author{
1,*Cirunay, A.R.T., ${ }^{1}$ Mopera, L.E., ${ }^{1}$ Sumague, M.J.V. and ${ }^{2}$ Bautista, J.A.N. \\ ${ }^{1}$ Institute of Food Science and Technology, College of Agriculture and Food Science, University of the \\ Philippines Los Baños, College, Laguna \\ ${ }^{2}$ Institute of Animal Science, College of Agriculture and Food Science, University of the Philippines Los \\ Baños, College, Laguna
}

\begin{abstract}
Article history:
Received: 24 July 2020

Received in revised form: 14 August 2020

Accepted: 21 September 2020

Available Online: 12

December 2020
\end{abstract}

Keywords:

Pigeon pea,

Prebiotic potential,

In vitro fermentation,

Resistant starch,

Dietary fiber

DOI:

https://doi.org/10.26656/fr.2017.5(1).388

\begin{abstract}
Prebiotics are widely recognized particularly for their role in selectively stimulating the growth and/or activity of beneficial bacteria in the gastrointestinal tract which beneficially affects the host health. Increasing interest has been given to the use of food materials that contain significant amounts of prebiotic components without the necessity of extracting these compounds. In this study, pigeon pea flour was evaluated as a potential prebiotic source. In vitro fermentation of pigeon pea flour by Lactobacillus plantarum (BIOTECH 1223) and Escherichia coli (BIOTECH 1634) was examined based on the changes in cell density, specific growth rate and mean doubling time of the microorganisms, as well as the change in total sugars, resistant starch, dietary fibers (insoluble, soluble and total), $\mathrm{pH}$, titratable acidity of the media. The prebiotic activity score of pigeon pea flour was also determined to measure the extent to which it encourages the selective growth of $L$. plantarum compared with that of $E$. coli under the same conditions. Results showed significantly higher growth and metabolic activity of L. plantarum than E. coli in modified medium containing pigeon pea flour. Prebiotic activity score of pigeon pea flour is 0.14 which is not significantly different from the 0.18 prebiotic activity score of commerciallyavailable inulin. Since the prebiotic activity score of pigeon pea flour is comparable to that of inulin, this activity can be extended to other commercially important probiotic organisms and can serve as a rational basis for identifying synbiotics for incorporation into various food products.
\end{abstract}

\section{Introduction}

Functional food products provide various health benefits to consumers by managing specific health conditions in a convenient way, particularly by means of their daily diet. Consumers have become increasingly reflective regarding health matters and willing to take on changes in their eating habits towards a more healthoriented diet (Niva, 2007). It is known that the balance of intestinal microbiota is important in human health, thus, significant efforts have been made to influence bowel flora through consumers' diet to beneficially affect the health of the host (Xiaoli et al., 2008). Much interest has been given to the idea of actively managing the intestinal microbiota for the purpose of the host's health improvement. Various opportunities for research have become available for the development of a wide range of new functional food concepts due to the increased awareness of the consumers coupled with the advances in numerous scientific domains.

Prebiotics are finding much-increased application into the food sector that provides a similar purpose to probiotics, particularly the improvement of the composition of the gut microflora community. The International Scientific Association of Probiotics and Prebiotics (ISAPP) defined dietary prebiotic as "a selectively fermented ingredient that results in specific changes in the composition and/or activity of the gastrointestinal microbiota, thus conferring benefit(s) upon host health" (Gibson et al., 2010).

Several studies on prebiotics have been carried out with the focus on purifying oligosaccharides from renewable resources and evaluating its effect on the composition of the gut microflora (Gullon et al., 2011; Gomez et al., 2014; Gullon et al., 2014). Nowadays, there is an increasing interest in the use of food materials 
that contain significant amounts of prebiotic components without the necessity of extracting these compounds.

Prebiotic carbohydrates can be found in various food crops. Legumes are potential sources of prebiotics that potentially modify the gut microbiota (Foyer et al., 2016). Pulses, which are the dry edible seeds of legumes, are known to have high amounts of protein, contain important vitamins and minerals, and have significant amounts of complex carbohydrates including oligosaccharides, resistant starch and dietary fibers. Thus, pulses can serve as an excellent source of growth factors and prebiotic components and can also be added to food products to possibly improve food formulation or create new functional food concepts.

Pigeon pea (Cajanus cajan (L.) Millsp.), locally known as kadios, is one of the underutilized indigenous legumes in the Philippines that has not been fully explored. This crop contains high amounts of carbohydrates, including starch, dietary fibers and oligosaccharides (Reddy et al., 1984). The major $\alpha$ galactooligosaccharides in grain legume seeds are raffinose, stachyose and verbascose. These oligosaccharides are not degraded in the upper gastrointestinal tract since humans lack $\alpha$-galactosidase, thus, they are fermented by colonic microflora, leading to the production of short-chain fatty acids and gases (Pratap and Kumar, 2011). A study conducted by Trinidad et al. (2010) showed the potential health benefits of legumes that are found in the Philippines, including pigeon pea which was reported as a good source of dietary fiber.

There is very limited information available on the prebiotic potential of pigeon pea. Thus, in this study, in vitro fermentation and prebiotic potential of pigeon pea flour were examined based on the changes in the cell density, specific growth rate and mean doubling time of the microorganisms, as well as the change in total sugars, resistant starch, dietary fibers (insoluble, soluble and total), $\mathrm{pH}$, titratable acidity of the media during fermentation.

\section{Materials and methods}

\subsection{Preparation of pigeon pea flour}

Mature pigeon pea seeds (Farmer's Variety: Bangluwan) acquired from Salcedo, Ilocos Sur, Philippines, were washed, soaked in potable water, dehulled and dried at $60^{\circ} \mathrm{C}$ for $16 \mathrm{hrs}$. Dried, dehulled seeds were ground using a pin mill and passed through a 100 -mesh sieve. The flour was then stored in a clean, airtight plastic container at room temperature until needed for analysis.

\subsection{In vitro fermentability of pigeon pea flour}

\subsubsection{Bacterial strains and culture preparation}

Lactobacillus plantarum strain (BIOTECH 1223) and Escherichia coli strain (BIOTECH 1634) obtained from the University of the Philippines Los Baños National Institute of Molecular Biology and Biotechnology (UPLB - BIOTECH) were used for this study. L. plantarum is well-characterized and established as probiotic while E. coli was selected to represent the enteric portion of the human gut microflora. The cultures were transferred onto de Man-Rogosa-Sharpe (MRS) agar for L. plantarum and nutrient agar for E. coli using streak plate method. The plates were incubated for 24 to $48 \mathrm{hrs}$ at $37^{\circ} \mathrm{C}$. A single colony from each plate was selected and transferred into $10 \mathrm{~mL}$ MRS broth for $L$. plantarum and $10 \mathrm{~mL}$ tryptic soy broth (TSB) for E. coli. The prepared cultures were then incubated at $37^{\circ} \mathrm{C}$ for 24 hrs and served as the seed cultures.

\subsubsection{Preparation of fermentation media}

Modified MRS broth and M9 minimal medium were used for L. plantarum and E. coli, respectively. MRS broth (devoid of glucose) and the M9 minimal medium were supplemented with $1.5 \%$ pigeon pea flour as main carbon source, which is the optimum concentration of pigeon pea flour that has a significant effect on the growth of $L$. plantarum according to Gerolaga (2018). Positive and negative controls were also prepared, wherein $1.5 \%$ inulin was used as the prebiotic carbon source for positive control.

\subsubsection{In Vitro Fermentation Assay}

The fermentation experiment was carried out using the method described by Liu et al. (2016) with modifications. Three separate fermentation experiments were carried out and conducted in triplicates using three treatments: negative control (basal medium - MRS broth and M9 minimal medium devoid of glucose), positive control (using commercial inulin, Orafti GR Inulin Fiber, as prebiotic carbohydrate source) and pigeon pea flour. Sterile fermentation media were then inoculated with $2 \%$ of 24-hour culture of each microorganism and incubated at $37^{\circ} \mathrm{C}$ for $48 \mathrm{hrs}$. Samples were taken at 0,24 and 48 hrs for enumeration of bacteria and for the determination of $\mathrm{pH}$, titratable acidity and total sugar. The viable counts of L. plantarum at 0,24 and $48 \mathrm{hrs}$ of incubation were determined using MRS agar while viable counts of E. coli were determined using Tryptic Soy Agar. Samples containing the pigeon pea flour were also analyzed to determine changes in the resistant starch and dietary fiber (total, soluble and insoluble) content of the medium. The growth rate $(\mu)$ and mean doubling time $\left(T_{d}\right)$ of the microorganisms were calculated using the 
following formula (Mansouri et al., 20156):

$$
\begin{gathered}
\mu=\frac{\ln x_{2}-\ln x_{1}}{t_{2}-t_{1}} \\
T_{d}=\frac{\ln 2}{\mu}
\end{gathered}
$$

where $\mathrm{x}_{2}$ and $\mathrm{x}_{1}$ are the cell densities of the microorganism at times $\mathrm{t}_{2}(24 \mathrm{hrs})$ and $\mathrm{t}_{1}(0 \mathrm{hr})$.

\subsubsection{Evaluation of Prebiotic Activity Score}

The prebiotic potential of the pigeon pea flour was then determined using the prebiotic activity assay described by Huebner et al. (2007). Using the obtained viable cell counts at 0 and $24 \mathrm{hrs}$ of incubation, the prebiotic activity score (PAS) was determined using the following equation:

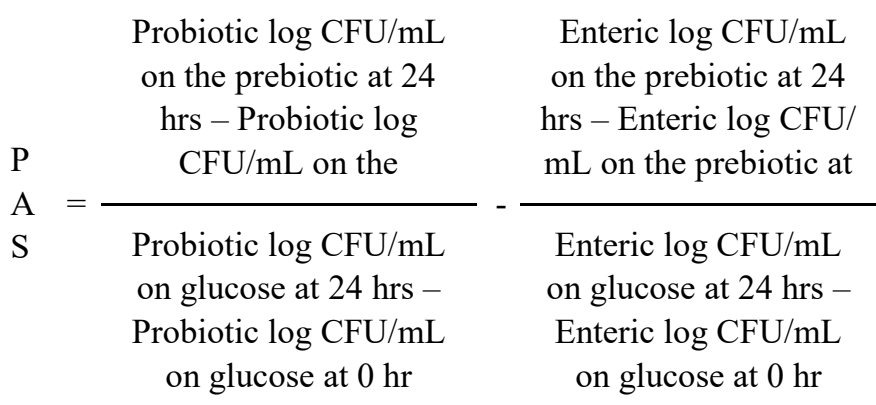

2.3 Determination of insoluble, soluble and total dietary fibers (Megazyme assay kit)

The dietary fiber (total, soluble and insoluble) content of the modified medium containing pigeon pea flour were determined using the Total Dietary Fiber Assay Procedure described by Megazyme (Megazyme International Ireland, 2016) which is based on AOAC Method 991.43 "Total, Soluble, and Insoluble Dietary Fiber in Foods" and AACC Method 32-07.01 "Determination of Soluble, Insoluble, and Total Dietary Fiber in Foods and Food Products".

\subsection{Determination of resistant starch (RS)}

The resistant starch content of the modified medium containing pigeon pea flour was determined using the Resistant Starch Assay Procedure by Megazyme (Megazyme Ireland Inc., 2015) which is accepted by AOAC Official Method 2002.02 and AACC Method 3240.01 .

\subsection{Physico-chemical Analyses}

The $\mathrm{pH}$ of samples was determined in triplicate using a $\mathrm{pH}$ pen. Total titratable acidity determination was performed and expressed as \% lactic acid. Total sugar was determined by Phenol-Sulfuric Acid Method (Dubois et al., 1956).

\subsection{Statistical Analysis}

All tests were conducted in triplicates. All experimental results were analyzed using analysis of variance (ANOVA). Test of significant differences among the treatment means was analyzed using the Tukey's Honesty Significant Difference (HSD). All statistical analyses were carried out using Statistica version 10 .

\section{Results and discussion}

\subsection{Effect of pigeon pea flour on the growth of microorganisms}

The cell densities of L. plantarum 1223 and E. coli 1634 in different treatments during a 48-hour fermentation period are summarized in Figures 1 and 2, respectively. The cell density of $L$. plantarum in the medium containing pigeon pea flour dramatically increased after $24 \mathrm{hrs}$ of incubation, after which a gradual increase was observed. There was a greater increase in the cell density of $L$. plantarum in the medium containing the flour compared to that of the negative control, but this increase was less than that of the positive control. Cell densities among treatments showed significant differences after 24 and $48 \mathrm{hrs}$ of incubation. The specific growth rate $\mu$ (per hour) and mean doubling time of $L$. plantarum in different treatments (Table 1) were also significantly different. Doubling time was used to determine the efficacy of different carbon sources to modulate the growth rate. Results revealed that the specific growth rate of $L$. plantarum was higher in the medium containing pigeon pea flour compared to the negative control but was less than the positive control. Conversely, the mean doubling time of the microorganism in the medium containing the pigeon pea flour is shorter than the negative control but longer than the positive control.

Table 1. Specific growth rate $(\mu)$ and mean doubling time $\left(T_{d}\right)$ of Lactobacillus plantarum in different treatments

\begin{tabular}{lcc}
\hline Treatment & $\mu$ (per hour) & $\mathrm{T}_{\mathrm{d}}$ \\
\hline Pigeon Pea Flour & $0.0099 \pm 0.0004^{\mathrm{b}}$ & $5.31 \pm 0.06^{\mathrm{b}}$ \\
*Positive Control $^{\mathrm{b}}$ & $0.0120 \pm 0.0012^{\mathrm{a}}$ & $5.12 \pm 0.10^{\mathrm{c}}$ \\
**Negative Control $^{\mathrm{c}}$ & $0.0088 \pm 0.0001^{\mathrm{c}}$ & $5.43+0.02^{\mathrm{a}}$ \\
\hline
\end{tabular}

Values are averages of three independent determinations \pm standard deviation

Values within a column with different superscripts are significantly different $(\mathrm{p}<0.05)$

*inulin as prebiotic carbon source

**MRS broth devoid of glucose

An increase in the cell density reflected the ability of bacteria to grow on the tested substrates. Pigeon pea flour was used in the modified medium to serve as the 


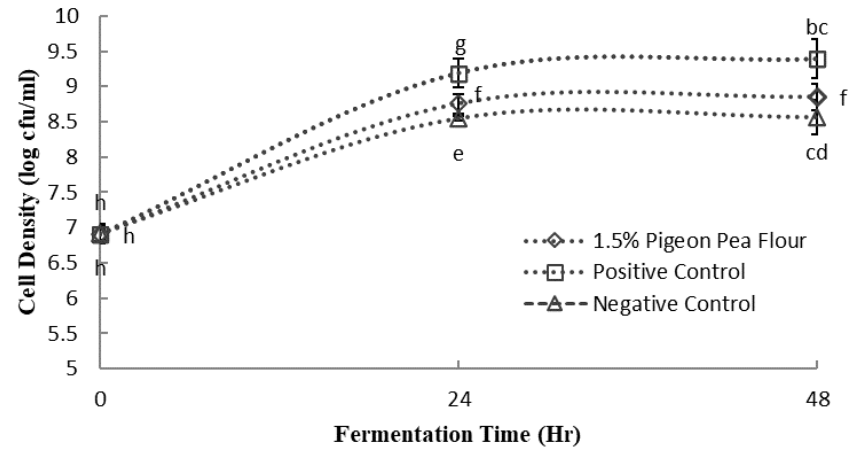

Figure 1. Cell density of Lactobacillus plantarum in different treatments during a 48 -hour fermentation process at $37^{\circ} \mathrm{C}$

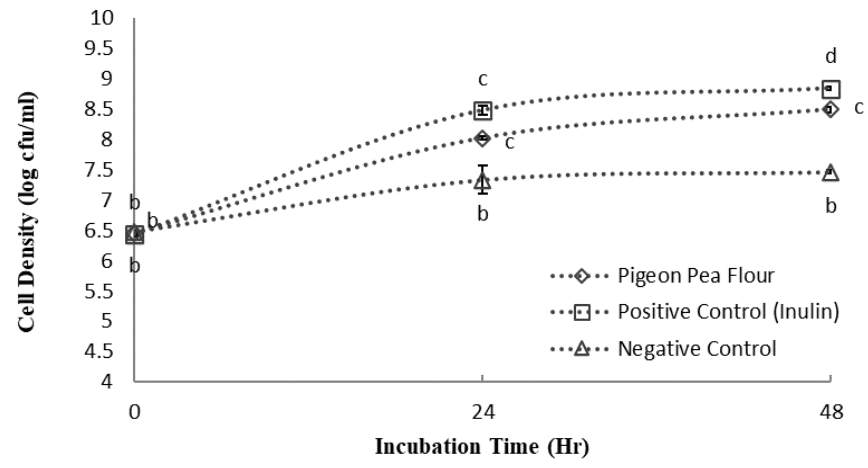

Figure 2. Cell density of Escherichia coli in different treatments during a 48 -hour fermentation process at $37^{\circ} \mathrm{C}$

main carbon source for the microorganism. Inulin was used as a positive control for its prebiotic effect and was reported as a good growth stimulant for probiotics. It has been found that inulin even at lower concentrations can encourage the selective growth of probiotic microorganisms (Donkor et al., 2007). Results showed that inulin had a greater effect on the growth of $L$. plantarum compared to pigeon pea flour, wherein the highest cell density and specific growth rate, as well as the shortest mean doubling time, were observed. Increase in the cell density of the microorganism in the medium containing pigeon pea flour was significantly different to that of the negative control, which indicates that the flour encouraged the growth of $L$. plantarum.

Microbial growth is dependent on the ability of the microorganism to use the available nutrients in the growth medium, including fermentable sugars (Parra et al., 2013). The changes in total sugar content of the modified MRS broth inoculated with $L$. plantarum are shown in Figure 3. A significant decrease in the total sugar content was observed in the medium containing pigeon pea flour, as well as in the positive control, throughout the incubation period. The reduction in the total sugar content of the medium containing pigeon pea flour means that $L$. plantarum was able to ferment and utilize the available simple sugars and carbohydrates for its growth, which is also observed in the increase in cell density of the microorganism, accompanied by a decrease in $\mathrm{pH}$ and increase in titratable acidity of the medium throughout the incubation period. Based on several studies, mature pigeon pea seeds contain a high amount of carbohydrates (Eltayeb et al., 2010; Olalekan and Bosede, 2010; Adamu and Oyetunde, 2013; Kunyanga et al., 2013; Oke, 2014). However, only a small portion of the total carbohydrates of pigeon pea is readily available for fermentation by microorganisms. Raw pigeon pea seeds contain small amounts of monosaccharides and reducing sugars. A large part of the total carbohydrates of pigeon pea is composed of starch and fibers, and the bulk sugars occurred as oligosaccharides which some microorganisms could not readily utilize. Generally, microorganisms ferment and utilize reducing sugars because these sugars have free carbon group that is essential for their growth and cultivation. Glucose is the major reducing sugar utilized during fermentation. Jairo et al. (1991) reported that pigeon pea seeds contain $0.14 \%$ glucose, $0.40 \%$ fructose and $4.02 \%$ sucrose while Apata (2008) reported that raw pigeon pea seeds contain $0.09-0.14 \%$ glucose, 0.29 $0.40 \%$ fructose and $2.01-2.25 \%$ sucrose. Raw pigeon pea seeds, having only small amounts of readily available carbohydrates for fermentation, may explain the slower growth of the microorganism on the medium compared to that of the positive control. As previously mentioned, a large part of the total carbohydrates is composed of dietary fibers and resistant starch, and the bulk of the sugars of pigeon pea seeds are oligosaccharides which are not readily utilized by some microorganisms. The major oligosaccharides present are the raffinose family oligosaccharides (RFOs), also known as $\alpha$-galactosides, which include stachyose, verbascose and raffinose. $L$. plantarum has the ability to produce $\alpha$-glucosidase enzyme that breaks down the $\alpha-1,6$-glycosidic bonds, which enables the microorganism to utilize the mentioned oligosaccharides (Duszkiewicz-Reinhard et al., 1994; Adewumi and Odunfa, 2009). However, the length of incubation in this study might not have been sufficient to determine whether L. plantarum was able to break down and fully utilize the oligosaccharides present in pigeon pea flour. A study conducted by Shimelis and Rakshit (2008) showed no significant difference in the concentration of $\alpha$-galactoside contents of several bean varieties (Phaseolus vulgaris L.) using controlled fermentation even after $96 \mathrm{hrs}$ of fermentation. Adewumi and Odunfa (2009) reported that the stachyose content of the two common Nigerian Vigna unguiculata beans inoculated with $L$. plantarum, $L$. fermentum and $P$. acidilactici was significantly reduced after incubation of samples for longer periods. It was also reported in the same study that the raffinose content of drum beans slurry was not significantly reduced when inoculated with $L$. plantarum and L. fermentum and incubated for $48 \mathrm{hrs}$. This is in comparison with the results of 
Czarnecka et al. (1998) who reported that raffinose content of bean seeds fermented with lactic acid bacteria was not reduced. This suggests that longer incubation period may be required in order for $L$. plantarum to be able to significantly reduce and utilize the oligosaccharides present in the medium.

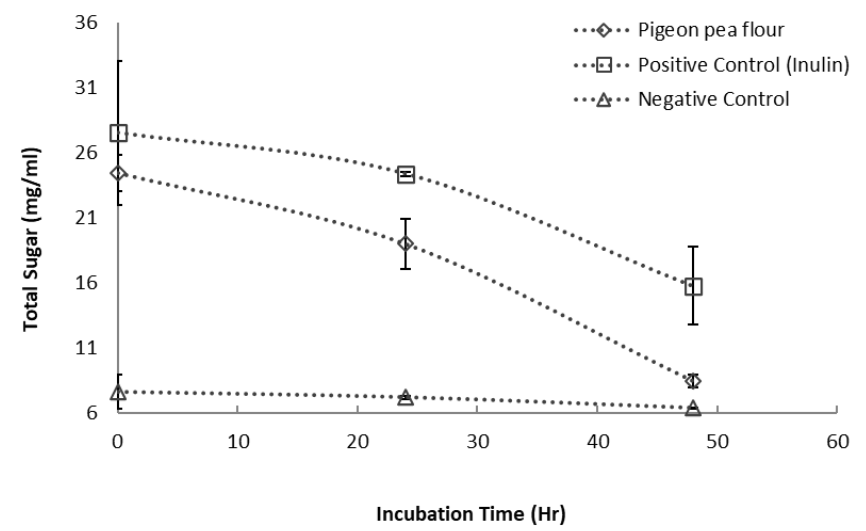

Figure 3. Total sugar $(\mathrm{mg} / \mathrm{mL})$ of modified MRS broth inoculated with Lactobacillus plantarum during the 48-hour fermentation period at 37

Table 2. Resistant starch content of the fermentation media with pigeon pea flour during the 48 -hour fermentation period at $37^{\circ} \mathrm{C}$

\begin{tabular}{ccc}
\hline \multirow{2}{*}{$\begin{array}{c}\text { Incubation } \\
\text { Time (Hr) }\end{array}$} & \multicolumn{2}{c}{ Resistant Starch (g/100 g) } \\
\cline { 2 - 3 } & $\begin{array}{c}\text { Modified MRS Broth }+ \text { Modified M9 medium } \\
\text { L. plantarum }\end{array}$ & + E. coli \\
\hline 0 & $0.43 \pm 0.11^{\mathrm{b}}$ & $0.43 \pm 0.12^{\mathrm{b}}$ \\
24 & $0.45 \pm 0.19^{\mathrm{b}}$ & $0.44 \pm 0.20^{\mathrm{b}}$ \\
48 & $0.54 \pm 0.05^{\mathrm{a}}$ & $0.54 \pm 0.09^{\mathrm{a}}$ \\
\hline
\end{tabular}

Values are averages of three independent determinations \pm standard deviation

Values within a column with different superscripts are significantly different $(\mathrm{p}<0.05)$

The RS and dietary fiber content of the modified MRS broth with pigeon pea flour were also measured to determine whether L. plantarum was able to break down and utilize the said complex carbohydrates during the fermentation period. The changes in the RS and dietary fiber (SDF, IDF and TDF) content of the fermentation medium are presented in Tables 2 and 3, respectively. Based on the results, there was no significant reduction in the RS content which means that $L$. plantarum was not able to break down and utilize the RS in the medium. An increase in the RS content was even observed towards the end of the fermentation period. On the other hand, significant changes in the dietary fiber components of the medium were observed. There was a significant increase in the SDF and a significant decrease in the IDF throughout the fermentation period. As mentioned in the earlier part of this study, fermentation results in the modifications on the composition and structure of the dietary fiber which are driven by the enzymatic activity that takes place during the fermentative process and these modifications primarily involve the solubilization of different cell wall polysaccharides. There was an observed significant reduction in the total dietary fiber of the medium towards the end of the fermentation period which means that a fraction of the dietary fiber was broken down by microbial and enzymatic action, and may have been fermented by L. plantarum.

On the other hand, the same trend was observed for the growth of $E$. coli in modified M9 medium. There was a significant increase in the cell density of $E$. coli in the medium containing pigeon pea flour after $24 \mathrm{hrs}$ of incubation. This increase was greater compared to the negative control but was significantly less compared to that of the positive control at $24 \mathrm{hrs}$ of incubation. After $48 \mathrm{hrs}$ of incubation, there was no significant increase in the cell density of the microorganism in the medium containing pigeon pea flour and in the negative control while a significant increase was observed in the positive control. The difference in the cell density is also reflected in the specific growth rate $\mu$ (per hour) and mean doubling time of $E$. coli in different treatments, wherein the specific growth rate $\mu$ (per hour) of the microorganism in the medium containing the flour was higher compared to the negative control, but was less than the positive control (Table 4). Conversely, the mean doubling time of $E$. coli in the medium containing the

Table 3. Dietary fiber content of fermentation media with pigeon pea flour during the 48 -hour fermentation period at $37^{\circ} \mathrm{C}$

\begin{tabular}{ccccc}
\hline \multirow{2}{*}{$\begin{array}{c}\text { Fermentation } \\
\text { Medium }\end{array}$} & $\begin{array}{c}\text { Incubation } \\
\text { Time (Hr) }\end{array}$ & \multicolumn{3}{c}{ Dietary Fiber (\%) } \\
\cline { 3 - 5 } & 0 & $0.96 \pm 0.04^{\mathrm{c}}$ & $1.24 \pm 0.05^{\mathrm{a}}$ & $2.20 \pm 0.07^{\mathrm{a}}$ \\
*Modified MRS & 24 & $1.02 \pm 0.04^{\mathrm{ab}}$ & $1.18 \pm 0.04^{\mathrm{b}}$ & $2.20 \pm 0.05^{\mathrm{a}}$ \\
Broth + L. plantarum & 48 & $1.07 \pm 0.04^{\mathrm{a}}$ & $1.04 \pm 0.04^{\mathrm{cd}}$ & $2.11 \pm 0.07^{\mathrm{b}}$ \\
\hline \multirow{2}{*}{ **Modified M9 } & 0 & $0.91 \pm 0.04^{\mathrm{d}}$ & $1.15 \pm 0.04^{\mathrm{b}}$ & $2.06 \pm 0.00^{\mathrm{b}}$ \\
medium + E. coli & 24 & $1.01 \pm 0.06^{\mathrm{bc}}$ & $1.08 \pm 0.04^{\mathrm{c}}$ & $2.08 \pm 0.08^{\mathrm{b}}$ \\
& 48 & $1.07 \pm 0.05^{\mathrm{a}}$ & $1.03 \pm 0.03^{\mathrm{d}}$ & $2.10 \pm 0.09^{\mathrm{b}}$ \\
\hline
\end{tabular}

Values are averages of three independent determinations \pm standard deviation

Values within a column with different superscripts are significantly different $(\mathrm{p}<0.05)$

*MRS broth (devoid of glucose) supplemented with pigeon pea flour as main carbon source

**M9 minimal medium (devoid of glucose) supplemented with pigeon pea flour as main carbon source 
pigeon pea flour is shorter than the negative control but longer than the positive control. There is a significant difference in the specific growth rate and mean doubling time of $E$. coli among treatments.

Table 4. Specific growth rate $(\mu)$ and mean doubling time (Td) of Escherichia coli in different treatments

\begin{tabular}{lcc}
\hline Treatment & $\mu$ (per hour) & $\mathrm{T}_{\mathrm{d}}$ \\
\hline Pigeon Pea Flour & $0.0090 \pm 0.00003^{\mathrm{b}}$ & $5.40 \pm 0.002^{\mathrm{b}}$ \\
$*$ Positive Control & $0.0115 \pm 0.0003^{\mathrm{a}}$ & $5.16 \pm 0.023^{\mathrm{c}}$ \\
**Negative Control $^{\mathrm{a}}$ & $0.0052 \pm 0.0012^{\mathrm{c}}$ & $5.96 \pm 0.240^{\mathrm{a}}$ \\
\hline
\end{tabular}

Values are averages of three independent determinations \pm standard deviation

Values within a column with different superscripts are significantly different $(\mathrm{p}<0.05)$

*inulin as prebiotic carbon source

**M9 minimal medium devoid of glucose

The increase in the cell density of $E$. coli in the medium showed that pigeon pea was able to support the growth of the microorganism, which means that E. coli was able to metabolize the available carbohydrates present in the medium. The changes in total sugar content of the modified M9 minimal medium inoculated with $E$. coli are shown in Figure 4. As for many other bacteria, the preferred carbon source of E. coli is glucose as this supports a faster growth rate of the microorganism compared to other sugars. In sugar mixtures, E. coli first metabolizes glucose, and when glucose is exhausted, shifts to grow more slowly on other sugars (Bren et al., 2016). The growth of the microorganism in the medium containing pigeon pea flour is in comparison with the results of Kafi et al. (2013) who reported that pigeon pea supported the growth of $E$. coli when used as a nutritional ingredient in culture media. The growth promotion of $E$. coli by inulin in this study is also similar to the results obtained by Mansouri et al. (2016) who observed an increased growth of $E$. coli when cultured in media supplemented with Jerusalem Artichoke fructans and standard prebiotic HP-inulin. In contrast, López-Molina et al. (2005) examined the use of chicory and Artichoke inulin (different degree of polymerization) in mixed cultures of colonic microorganisms and reported slower but longerlasting growth of $E$. coli in the culture media containing both inulins in comparison to the control medium containing glucose.

The analysis of the total sugar content of the different modified M9 media showed a significant decrease in total sugar of the medium containing pigeon pea flour, as well as the positive control, throughout the incubation period. The reduction in the total sugar content of the medium containing pigeon pea flour means that $E$. coli was able to ferment and utilize the available sugars and carbohydrates for its growth, which is also observed in the increase in the cell density of the microorganism. However, it is uncertain whether E. coli was able to utilize the oligosaccharides present in the medium since the reduction in the total sugar content of the fermentation medium was less compared to $L$. plantarum, which was also reflected on its slower growth rate. Similar to L. plantarum, the growth of E. coli on the modified medium containing pigeon pea flour was not due to RS, since there was no significant reduction in the RS content of the medium which means that it was not fermented and utilized by the microorganism. The same trend is also true for dietary fiber. Although significant modifications in the dietary fiber components (SDF and IDF) of the medium were observed, there was no significant reduction in the TDF content.

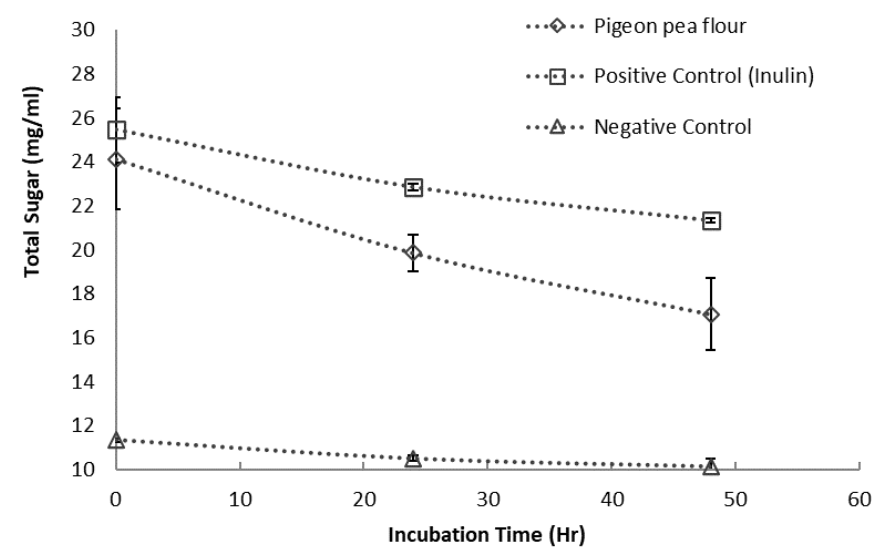

Figure 4. Total sugar $(\mathrm{mg} / \mathrm{mL})$ of modified M9 medium inoculated with Escherichia coli during the 48-hour fermentation period at $37^{\circ} \mathrm{C}$

Comparing the growth of the two microorganisms in the different fermentation treatments, there was a higher change in cell density of $L$. plantarum in the medium containing pigeon pea flour compared to E. coli. Higher specific growth rate and shorter mean doubling time were also observed in L. plantarum. The change in the cell densities of the two microorganisms, as well as their specific growth rates and mean doubling time, were found to be significantly different which suggest that pigeon pea flour has a greater positive effect on the growth of $L$. plantarum than that of E. coli.

\section{$3.2 \mathrm{pH}$ and titratable acidity}

The changes in $\mathrm{pH}$ and titratable acidity of the different fermentation media during the 48-hour fermentation period are presented in Figures 5 and 6 for L. plantarum and E. coli, respectively. After $24 \mathrm{hrs}$ of incubation, there was a significant decrease in the $\mathrm{pH}$ values of the fermentation medium containing the pigeon pea flour inoculated with L. plantarum. However, the $\mathrm{pH}$ drop of the medium is greater in the positive control compared to that containing the pigeon pea flour. A gradual decrease in $\mathrm{pH}$ was observed in both the positive 
control and the medium containing the pigeon pea flour after $48 \mathrm{hrs}$ of incubation while that of the negative control remained constant. On the other hand, an increase in the incubation time resulted in a significant increase in the titratable acidity of the fermentation media. However, the increase in the titratable acidity of the medium containing pigeon pea as the sole carbon source is lower compared to the positive control.

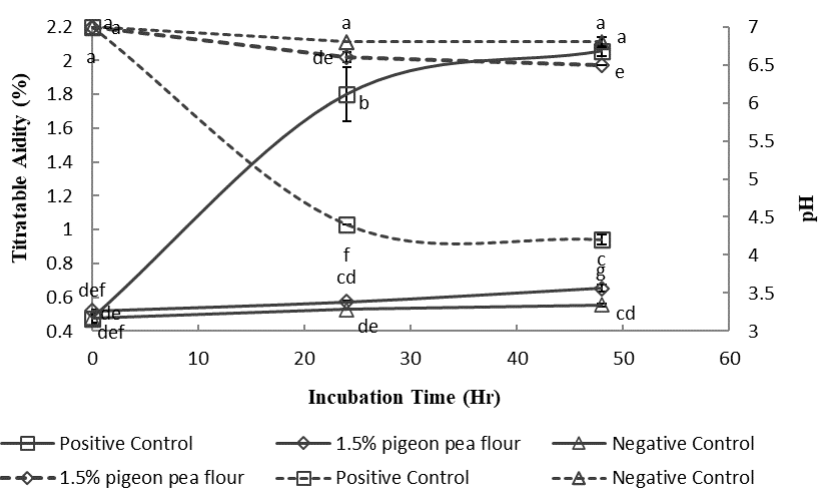

Figure 5. pH (dotted lines) and titratable acidity (solid lines) of modified MRS broth inoculated with Lactobacillus plantarum during the 48 -hour fermentation period at $37^{\circ} \mathrm{C}$

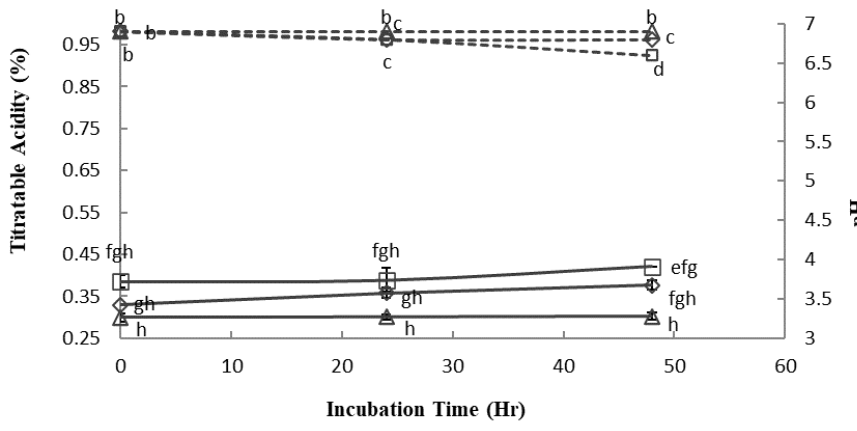

$\square$ Positive Control (Inulin) $\neg$ Pigeon pea flour $\quad \backsim$ Negative Control $-\leadsto--$ Pigeon pea flour $\quad--\square--$ Positive Control (Inulin) --A-- Negative Control

Figure 6. $\mathrm{pH}$ (broken lines) and titratable acidity (solid lines) of modified M9 medium inoculated with Escherichia coli during a 48 -hour fermentation period at $37^{\circ} \mathrm{C}$

Over the course of the process of fermentation, lactic acid is produced by lactic acid bacteria from carbohydrates, such as hexoses, oligosaccharides and complex sugars (Doblado et al., 2003). The acid produced during fermentation leads to the decrease in the $\mathrm{pH}$ of the media, thus, is used as an indirect measure of the efficiency of the fermentation process (Granito et al., 2003). L. plantarum is a facultative heterofermentative lactobacillus that has the ability to produce $\alpha$-glucosidase which is an enzyme capable of breaking down the $\alpha-1,6-$ glycosidic bonds of carbohydrates (Adewumi and Odunfa, 2009).

Raw pigeon pea flour having small amounts of monosaccharides and reducing sugars which are available for fermentation by $L$. plantarum explains the slower drop in $\mathrm{pH}$ and increase in titratable acidity of the medium containing pigeon pea flour compared to that of the positive control. Inulin, on the other hand, is a prebiotic substrate which means that it is selectively fermented by probiotic microorganisms (Sharma and Kanwar, 2017). There is a drastic drop in the $\mathrm{pH}$ of the positive control after $24 \mathrm{hrs}$ of incubation which means that inulin was fermented by L. plantarum, resulting in the increase in titratable acidity due to the organic acids produced. This is in agreement with the results reported by Sharma and Kanwar (2017) wherein a rapid drop in $\mathrm{pH}$ was observed in the in vitro fermentation of inulin using L. plantarum. The difference in the change in $\mathrm{pH}$ of the medium containing inulin may be due to the difference in strains of $L$. plantarum used in the in vitro assay. Thus, the fermentation process is more efficient in the positive control as reflected in the rapid $\mathrm{pH}$ drop and increase in the titratable acidity of the medium after 24 and $48 \mathrm{hrs}$ of incubation.

The reduction in the $\mathrm{pH}$ values and increase in the titratable acidity of the medium containing pigeon pea flour is in comparison with the results of Adewumi and Odunfa (2009) who reported a decrease in the $\mathrm{pH}$ and increase in titratable acidity during controlled fermentation of beans (Vigna unguiculata) with $L$. plantarum, L. fermentum and $P$. acidilactici from 24 to $72 \mathrm{hrs}$. This is also in agreement with the findings of E Granito et al. (2003) who reported a similar trend in controlled fermentation of beans (Phaseolus vulgaris) from 24 to $72 \mathrm{hrs}$ using Lactobacillus acidophilus, Bifidobacterium and Streptococcus thermophilus.

After $24 \mathrm{hrs}$ of fermentation with E. coli, a gradual decrease in $\mathrm{pH}$ was observed in both the positive control and the medium containing the pigeon pea flour. The $\mathrm{pH}$ of the medium containing the flour then remained unchanged after $48 \mathrm{hrs}$. On the other hand, a gradual drop in the $\mathrm{pH}$ of the positive control was observed after the 48-hour fermentation period. The $\mathrm{pH}$ of the negative control remained unchanged throughout the fermentation time. There is also a corresponding increase in the titratable acidity for both positive control and the medium containing the pigeon pea flour after $48 \mathrm{hrs}$ of fermentation while that of the negative control remained constant.

Comparing the $\mathrm{pH}$ and titratable acidity changes of the medium containing pigeon pea flour as fermented by L. plantarum and E. coli, a more rapid $\mathrm{pH}$ drop and a greater increase in titratable acidity was observed in the medium fermented by $L$. plantarum. There is a significant difference in the $\mathrm{pH}$ and titratable acidity values between the two treatments throughout the fermentation time. This would mean that pigeon pea flour was more efficiently fermented by $L$. plantarum than E. coli. 


\subsection{Prebiotic activity score}

Prebiotics are known to have the ability to influence the population of the colonic microbiota because of their selective utilization. The growth of microorganisms capable of rapidly fermenting prebiotics is encouraged, at the expense of those that are incapable of prebiotic fermentation. Thus, the effectiveness of prebiotic carbohydrates is dependent on the selective fermentation and enhanced growth of the specific targeted microorganisms. In addition, a prebiotic substrate should not be fermented by commensal organisms (Huebner et al., 2007). This study investigated the prebiotic effect of pigeon pea flour using pure culture fermentation. The extent to which pigeon pea flour expresses prebiotic activity was quantified. In order for a carbohydrate to have prebiotic activity, a test strain should be able to metabolize this substrate as well, or nearly as well, as the microorganism metabolizes glucose (Huebner et al., 2007). In this study, the prebiotic activity scores of the two substrates (pigeon pea flour and inulin) were computed using the cell densities of L. plantarum and $E$. coli on glucose reported by Ortiguero (2014). The author reported an increase of $2.29 \log \mathrm{CFU} / \mathrm{mL}$ in the cell density of L. plantarum and $2.26 \log \mathrm{CFU} / \mathrm{mL}$ in the cell density of $E$. coli after $24 \mathrm{hrs}$ of incubation with glucose. The computed prebiotic activity scores for pigeon pea flour and inulin are presented in Table 5.

Table 5. Prebiotic activity scores of inulin and pigeon pea flour

\begin{tabular}{lc}
\hline Treatment & Prebiotic Score \\
\hline Inulin & $0.18 \pm 0.04^{\mathrm{a}}$ \\
Pigeon Pea Flour & $0.14 \pm 0.08^{\mathrm{a}}$ \\
\hline
\end{tabular}

Values are averages of three independent determinations \pm standard deviation

Values within a column with different superscripts are significantly different $(\mathrm{p}<0.05)$.

Results showed that inulin had higher prebiotic activity score compared to pigeon pea flour; however, the scores were not significantly different $(\mathrm{P}<0.05)$. When compared with the well-known prebiotic inulin, pigeon pea flour was found to have a comparable effect on the two microorganisms. The computed prebiotic activity scores were low since the growth of the test strain on the prebiotic substrates was less (based on cell densities) compared with that on glucose. A considerable variation in the prebiotic activity scores may be observed for the different prebiotics utilized by a single probiotic strain since it is known that lactobacilli are metabolically diverse. Huebner et al. (2007) reported significant differences in the prebiotic activity scores of a strain of L. plantarum for different commercial prebiotics. According to this author, there exists a difference in the metabolic capacity of related strains as indicated by the significant difference in prebiotic scores even of strains within a single species. This explains the difference in the prebiotic activity scores for inulin reported by Huebner et al. (2007) as compared to the prebiotic score obtained in this study. The author reported a higher prebiotic activity score of $L$. plantarum 4008 for inulin compared to the results in this study, while L. plantarum 12006 had a negative prebiotic activity score. Based on several studies, presence of specific hydrolysis and transport systems is required for a particular prebiotic to be utilized by lactic acid and related bacteria (Gopal, et al., 2001; Perrin, et al., 2001; Rabiu, et al., 2001; Barrangou et al., 2003; Kaplan and Hutkins, 2003). Thus, variations in the prebiotic activity scores are possible depending on the presence or absence of genes coding for these metabolic systems.

\section{Conclusion}

Pigeon pea flour encouraged the growth of $L$. plantarum 1223 with cell density higher than that of $E$. coli 1634 . The metabolic activity of $L$. plantarum was also stimulated by the pigeon pea flour, as there was a more rapid $\mathrm{pH}$ drop in the medium fermented by $L$. plantarum compared to that of E. coli. The $\mathrm{pH}$ and titratable acidity values of the modified MRS broth and modified M9 medium throughout the fermentation time were found to be significantly different, which means that pigeon pea flour was more efficiently fermented by $L$. plantarum than $E$. coli. The increased cell density and metabolic activity of L. plantarum may be attributed to its utilization of the oligosaccharides and dietary fibers present in the pigeon pea flour. The prebiotic activity scores measured in this study determines the extent to which pigeon pea flour would promote selective growth of $L$. plantarum. Since the prebiotic potential of pigeon pea flour is comparable to that of inulin, this activity can be extended to other commercially important probiotic organisms and can serve as a rational basis for identifying synbiotics for incorporation into various food products.

\section{Conflict of interest}

All authors declare no conflict of interest.

\section{Acknowledgments}

The authors would like to thank the Department of Science and Technology - Science Education Institute Accelerated Science and Technology Human Resource Development Program for funding this research. 


\section{References}

Adamu, A.S. and Oyetunde, J.G. (2013). Comparison of dietary proximate and mineral values of two varieties of bean. Asian Journal of Natural and Applied Sciences, 2(2), 103-106.

Adewumi, G.A. and Odunfa, S.A. (2009). Effect of controlled fermentation on the oligosaccharides content of two common Nigerian Vigna unguiculata beans (drum and oloyin). African Journal of Biotechnology, 8(11), 2626-2630.

Apata, D.F. (2008). Effect of cooking methods on available and unavailable carbohydrates of some tropical grain legumes. African Journal of Biotechnology, 7(16), 2940-2945.

Barrangou, R., Altermann, E., Hutkins, R., Cano, R. and Klaenhammer, T.R. (2003). Functional and comparative genomic analyses of an operon involved in fructooligosaccharide utilization by Lactobacillus acidophilus. Proceedings of the National Academy of Sciences, 100, 8957-8962. https://doi.org/10.1073/ pnas. 1332765100

Bren, A., Park, J.O., Towbin, B.D., Dekel, E., Rabinowitz, J.D. and Alon, U. (2016). Glucose becomes one of the worst carbon sources for $E$. coli on poor nitrogen sources due to suboptimal levels of cAMP. Scientific Reports, 6, 24834. https:// doi.org/10.1038/srep24834

Czarnecka, M., Czarnecki, Z., Nowak, J. and Roszyk, H. (1998). Effect of lactic fermentation and extrusion of bean and pea seeds on nutritional and functional properties. Die Nahrung, 42(1), 7-11. https:// doi.org/10.1002/(SICI)1521-3803(199802) 42:01<7::AID-FOOD7>3.3.CO;2-9

Doblado, R., Frias, J., Muñoz, R. and Vidal-Valverde, C. (2003). Fermentation of Vigna sinensis var. carilla Flours by Natural Microflora and Lactobacillus Species. Journal of Food Protection, 66(12), 23132320. https://doi.org/10.4315/0362-028X-66.12.2313

Donkor, O.N., Nilmini, S.L.I., Stolic, P., Vasiljevic, T. and Shah, N.P. (2007). Survival and activity of selected probiotic organisms in set-type yoghurt during cold storage. International Dairy Journal, 17 (6), 657-665. https://doi.org/10.1016/ j.idairyj.2006.08.006

Dubois, M., Gilles, K.A., Hamilton, J.K., Rebers, P.A. and Smith, F. (1956). Colorimetric Method for Determination of Sugars and Related Substances. Analytical Chemistry, 28(3), 350-356. https:// doi.org/10.1021/ac60111a017

Duszkiewicz-Reinhard, W., Gujska, E. and Khan, K. (1994). Reduction of stachyose in legume flours by lactic acid bacteria. Journal of Food Science, 59(1),
115-117.

https://doi.org/10.1111/j.1365-

2621.1994.tb06911.x

Eltayeb, A.R.S.M., Ali, A.O. and Haron, R. (2010). The chemical composition of pigeon pea (Cajanus cajan) seed and functional properties of protein isolate. Pakistan Journal of Nutrition, 9(11), 1069-1073. https://doi.org/10.3923/pjn.2010.1069.1073

Foyer, C.H., Lam, H.M., Nguyen, H.T., Siddique, K.H.M., Varshney, R.K., Colmer, T.D., Cowling, W., BramLey, H., Mori, T.A., Hodgson, J.M., Cooper, J.W, Miller, A.J., Kunert, K., Vorster, J., Cullis, C., Ozga, J.A., Wahlqvist, M.L., Liang, Y., Shou, H., Shi, K., Yu, J., Fodor, N., Kaiser, B.N., Wong, F.L., Valliyodan, B. and Considine, M.J. (2016). Neglecting legumes has compromised human health and sustainable food production. Nature Plants, 2, 16112. https://doi.org/10.1038/ nplants.2016.112

Gerolaga, P.J.R. (2018). Multiple Response Optimization of the Growth Conditions of Lactobacillus plantarum using Pigeon Pea (Cajanus Cajan) Flour as a Substitute Carbon Source. Philippines: University of the Philippines Los Baños, BSc. Thesis

Gibson, G.R., Scott, K.P., Rastall, R.A., Tuohy, K.M., Hotchkiss, A., Dubert-Ferrandon, A., Gareau, M., Murphy, E.F., Saulnier, D., Loh, G., Macfarlane, S., Delzenne, N., Ringel, Y., Kozianowski, G., Dickmann, R., Lenoir-Wijnkook, I., Walker, C. and Buddington, R. (2010). Dietary prebiotics: current status and new definition. Food Science and Technology Bulletin: Functional Foods, 7(1), 1-19. https://doi.org/10.1616/1476-2137.15880

Gomez, B., Gullon, B., Remoroza, C., Schols, H.A., Parajo, J.C. and Alonso, J.L. Purification, Characterization and Prebiotic Properties of Pectic Oligosaccharides from Orange Peel Wastes. (2014). Journal of Agricultural and Food Chemistry, 62(40), 9769-82. https://doi.org/10.1021/jf503475b

Gopal, P.K., Sullivan, P.A. and Smart, J.B. (2001). Utilisation of galactooligosaccharides as selective substrates for growth by lactic acid bacteria including Bifidobacterium lactis DR10 and Lactobacillus rhamnosus DR20. International Dairy Journal, 11, 19-25. https://doi.org/10.1016/S09586946(01)00026-7

Granito, M., Champ, M., Guerra, M. and Frias, J. (2003). Effect of natural and controlled fermentation on flatus-producing compounds of beans (Phaseolus vulgaris). Journal of the Science of Food and Agriculture, 83(10), 1004-1009. https:// doi.org/10.1002/jsfa.1388

Gullon, B., Gullon, P. Sanz, Y., Alonso, J.L. and Parajo, J.C. (2011). Prebiotic potential of a refined product 
containing pectic oligosaccharides. LWT - Food Science and Technology, 44(8), 1687-1696. https:// doi.org/10.1016/j.lwt.2011.03.006

Gullon, B., Gullon, P., Tavaria, F. Pintado, M. Gomes, A.M. Alonzo, J.L. and Parajo, J.C. (2014). Structural features and assessment of prebiotic activity of refined arabinoxyloologosaccharides from wheat bran. Journal of Functional Foods, 6, 438-449. https://doi.org/10.1016/j.jff.2013.11.010

Huebner, J., Wehling, R.L. and Hutkins, R.W. (2007), Functional activity of commercial prebiotics. International Dairy Journal, 17(7), 770-775. https:// doi.org/10.1016/j.idairyj.2006.10.006

Jairo, O.C., Hugo, C.S., Gilberto, L.B. and De Roberto, C. (1991). Oligosaccharide levels in immature and mature seeds from several varieties of pigeon peas (Cajanus cajan). Alimentos Nutricaoararaquara, 3, 75-85.

Kafi, S.K., Mohana, R., Daniel, Shokri, M.D. and Musa, H.A. (2013). Use of Cowpea and Pigeon pea as Nutritional Ingredients in Culture Media. Journal of Medical Sciences, 8(3), 141-146.

Kaplan, H. and Hutkins, R.W. (2003). Metabolism of fructooligosaccharides by Lactobacillus paracasei 1195. Applied and Environmental Microbiology, 69, 2217-2222. https://doi.org/10.1128/AEM.69.4.22172222.2003

Kunyanga, C., Imungi, J. and Vellingiri, V. (2013). Nutritional evaluation of indigenous foods with potential food-based solution to alleviate hunger and malnutrition in Kenya. Journal of Applied Bioscience, 67, 5277-5288. https://doi.org/10.4314/ jab.v67i0.95049

López-Molina, D., Navarro-Martínez, M.D., RojasMelgarejo, F., Hiner, A.N.P., Chazarra, S. and Rodríguez-López, J.N. (2005). Molecular properties and prebiotic effect of inulin obtained from artichoke (Cynara scolymus L.). Phytochemistry, 66(12), 1476 -1484 .

j.phytochem.2005.04.003

Liu, Y., Gibson, G.R. and Walton, G.E. (2016). An In Vitro Approach to Study Effects of Prebiotics and Probiotics on the Faecal Microbiota and Selected Immune Parameters Relevant to the Elderly. PLoS ONE, 11(9), e0162604. https://doi.org/10.1371/ journal.pone. 0162604

Mansouri, E., Milani, E., Sani, A.M. and Nourbakhsh, L. (2016). Viability of Bifidobacterium bifidum and Escherichia coli in Versus Prebiotic Effects of Jerusalem Artichoke (Helianthus tuberosus). Zahedan Journal of Research in Medical Sciences, 18(11), e3771. https://doi.org/10.17795/zjrms-3771
Megazyme Ireland International. (2016). Total Dietary Fiber Assay Procedure. Retrieved from Megazyme website: https://www.megazyme.com/documents/ Booklet/K-TDFR-200A_DATA.pdf

Megazyme Ireland International. (2015). Resistant Starch Assay Procedure. Retrieved from Megazyme website: https://www.megazyme.com/documents/ Booklet/K-RAPRS_DATA.pdf

Niva, M. (2007). All foods affect health: Understandings of functional foods and healthy eating among healthoriented Finns. Appetite, 48(3), 384-393. https:// doi.org/10.1016/j.appet.2006.10.006

Oke, D.G. (2014). Proximate and phytochemical analysis of Cajanus cajan (Pigeon Pea) leaves. Chemical Science Transactions, 3(3), 1172-1178.

Olalekan, A.J. and Bosede, B.F. (2010). Comparative study on chemical composition and functional properties of three Nigerian legumes (Jack beans, Pigeon pea and Cowpea). Journal of Emerging Trends in Engineering and Applied Sciences, 1(1), 89-95.

Ortiguero, M.C.G. (2014). Evaluation of Prebiotic Potential of Sweet Potato (Ipomoea Batatas L.) through In Vitro Assay. Philippines: University of the Philippines Los Baños, BSc. Thesis

Parra, K., Ferrer, M., Piñero, M., Barboza, Y. and Medina, L.M. (2013). Use of Lactobacillus acidophilus and Lactobacillus casei for a Potential Probiotic Legume-Based Fermented Product Using Pigeon Pea (Cajanus cajan). Journal of Food Protection, 76(2), 265-271. https:// doi.org/10.4315/0362-028X.JFP-12-138

Perrin, S., Warchol, M., Grill, J.P. and Schneider, F. (2001). Fermentations of fructo-oligosaccharides and their components by Bifidobacterium infantis ATCC 15697 on batch culture in semi-synthetic medium. Journal of Applied Microbiology, 90(6), 859-865. https://doi.org/10.1046/j.1365-2672.2001.01317.x

Pratap, A. And Kumar, J. (2011). Biology and Breeding of Food Legumes. UK: CABI International. https:// doi.org/10.1079/9781845937669.0000

Rabiu, B.A., Jay, A.J., Gibson, G.R. and Rastall, R.A. (2001). Synthesis and fermentation properties of novel galacto-oligosaccharides by $\beta$-galactosidases from Bifidobacterium species. Applied and Environmental Microbiology, 67, 2526-2530. https://doi.org/10.1128/AEM.67.6.2526-2530.2001

Reddy, N.R., Pierson, M.D., Sathe, S.K. and Salunkhe, D.K. (1984). Chemical, nutritional and physiological aspects of dry bean carbohydrates: a review. Food Chemistry, 13(1), 25-68. https:// doi.org/10.1016/0308-8146(84)90026-8 
Sharma, S. and Kanwar, S.S. (2017). Effect of prebiotics on growth behavior of Lactobacillus plantarum and their impact on adherence of strict anaerobic pathogens to intestinal cell lines. Journal of Food Safety, 38(1), e12384. https://doi.org/10.1111/ jfs. 12384

Shimelis, E.A. and Rakshit, S.K. (2008). Influence of natural and controlled fermentations on $\alpha$ galactosides, antinutrients and protein digestibility of beans (Phaseolus vulgaris L.). International Journal of Food Science and Technology, 43(4), 658-665. https://doi.org/10.1111/j.1365-2621.2006.01506.x

Trinidad, T.P., Mallillin, A.C., Loyola, A.S., Sagum, R.S. and Encabo, R.R. (2009). The potential health benefits of legumes as a good source of dietary fibre. British Journal of Nutrition, 103(4), 569-574. https:// doi.org/10.1017/S0007114509992157

Xiaoli, X. Liyi, Y., Shuang, H. Wei, L. Yi, S. Hao, M. Jusong, Z. and Xiaoxiong, Z. (2008). Determination of oligosaccharide contents in 19 cultivars of chickpea (Cicer arietinum L) seeds by high performance liquid chromatography. Food Chemistry, 111(1), 215-219. https://doi.org/10.1016/ j.foodchem.2008.03.039 